Annals of Pure and Applied Mathematics

Vol. 17, No. 1, 2018, 23-28

ISSN: 2279-087X (P), 2279-0888(online)

Published on 11 April 2018

www.researchmathsci.org

DOI: http://dx.doi.org/10.22457/apam.v17n1a3

Annals of

Pure and Applied

Mathematics

\title{
Convergence of Interpolatory Polynomial Between Lagrange and Hermite
}

\author{
Swarnima Bahadur ${ }^{1}$ and Varun ${ }^{2}$ \\ ${ }^{1}$ Department Of Mathematics and Astronomy, University of Lucknow \\ Lucknow-226007, U.P, India. \\ E-mail: ${ }^{1}$ swarnimabahadur@ymail.com; ${ }^{2}$ varun.kanaujia.1992@ gmail.com \\ ${ }^{2}$ Corresponding author
}

Received 1 March 2018; accepted 9 April 2018

\begin{abstract}
The aim of this paperis to study an interpolation problem, which is an intermediate problem between Lagrange and Hermite. We consider this problem on the nodes obtained by projecting vertically the zeros of $\left(1-x^{2}\right) P_{n}(x)$ onto the unit circle, where $P_{n}(x)$ stands for $n^{\text {th }}$ Legendre polynomial. We prove the regularity of the problem, give explicit forms and establish a convergence theorem for the same.
\end{abstract}

Keywords: Legendre polynomial, Explicit representation, Convergence

AMS Mathematics Subject Classification(2010): 41A05, 30E10

\section{Introduction}

In 1990, Tian Liang Tu [7] obtained the divergence and mean convergence of the Hermite interpolation operator. In 1993, Prasad [6] studied the Hermite-Fejer interpolation of higher order on the n-distinct zeros of $\left(1-x^{2}\right) P_{n-2}(x)$, where $P_{n}(x)$ is the $n^{\text {th }}$ Legendre polynomial. In 1998, Bahadur and Mathur [3] proved the convergence of Quasi-Hermite interpolation on the nodes obtained by projecting vertically the zeros of $\left(1-x^{2}\right) P_{n}(x)$ onto the unit circle, where $P_{n}(x)$ is the $n^{\text {th }}$ Legendre polynomial. In 2001, Daruls and Gonzalezvera [4] gave an extension to the unit circle of the classical Hermite-Fejer Theorem. In 2011, Bahadur [1] presented a method for computing the convergence of Hermite interpolation polynomial onto the unit circle.

In 2016, Bahadur and Bano [2] considered modified Hermite interpolation onto the unit circle. Later on, several mathematician have considered Hermite and Lagrange interpolation on different set of nodes. These have motivated us to consider a problem between Lagrange and Hermite interpolation.

In this paper, we consider an interpolation problem on the nodes, which are obtained by projecting vertically the zeros of $\left(1-x^{2}\right) P_{n}(x)$ onto the unit circle. We prescribe the functional value at \pm 1 , whereas first derivative at all other points.

In section 2 , we give some preliminaries and in section 3 , we describe the problem and give the existence theorem of the interpolatory polynomials, whereas in section 4, we give the explicit formulae of theinterpolatory polynomials. Lastly in section 5 and 6, we give estimates and convergence ofinterpolatory polynomials respectively. 


\section{Preliminaries}

In this section, we shall give some well knownresults.

$\left\{z_{0}=1, z_{2 n+1}=-1, z_{k}=\cos \theta_{k}+i \sin \theta_{k}, z_{n+k}=-z_{k}, k=1(1) n\right\}$

be the vertical projections on unit circle of the zeroes of $\left(1-x^{2}\right) P_{n}(x)$, where $P_{n}(x)$ stands for $n^{\text {th }}$ Legendre polynomial having zeros,

$$
\begin{gathered}
x_{k}=\cos \theta_{k}, k=1(1) n \text { s.t. } \quad 1>x_{1}>x_{2}>\cdots>x_{n}>-1 \\
\mathrm{~W}(\mathrm{z})=\prod_{k=1}^{2 n}\left(z-z_{k}\right)=K_{n} P_{n}\left(\frac{1+z^{2}}{2 z}\right) z_{n} \\
K_{n}=\frac{\left(2^{n} n !\right)}{(2 n-1) ! !} \\
R(z)=\left(z^{2}-1\right) W(z)
\end{gathered}
$$

The differential equation satisfied by $P_{n}(x)$ is

$$
\left(1-x^{2}\right) P_{n}^{\prime \prime}(x)-2 x P_{n}^{\prime}(x)+n(n+1) P_{n}(x)=0
$$

Fundamental polynomials of Lagrange interpolation based on the zeros of $W(z)$ and $R(z)$ are given by

$$
\begin{gathered}
L_{1 k}(z)=\frac{R(z)}{\left(z-z_{k}\right) R^{\prime}\left(z_{k}\right)} k=0(1) 2 n+1 \\
L_{k}(z)=\frac{W(z)}{\left(z-z_{k}\right) W^{\prime}\left(z_{k}\right)} k=1(1) 2 n
\end{gathered}
$$

For $-1 \leq x \leq 1$ we have,

$$
\begin{aligned}
& \left|z^{2}-1\right|=2 \sqrt{1-x^{2}} \\
& \quad\left(1-x^{2}\right)^{\frac{1}{4}}\left|P_{n}(x)\right| \leq \sqrt{\frac{2}{\pi n}} \\
& \left|P_{n}(x)\right| \leq 1
\end{aligned}
$$

Let $x_{k}^{\prime} s$ be the zeroes of $P_{n}(x)$, then

$$
\begin{gathered}
\left(1-x_{k}^{2}\right)^{-1} \sim\left(\frac{k}{n}\right)^{-2} \\
\left|P_{n}^{\prime}\left(x_{k}\right)\right| \geq c k^{-\frac{3}{2}} n^{2}
\end{gathered}
$$

\section{The problem and the regularity}

Let $Z_{n}=\left\{z_{k} ; k=0(1) 2 n+1\right\}$ satisfying (2.1),

Here, we are interested in determining the interpolatory polynomial $L_{n}(z)$ of degree $\leq$ $2 \mathrm{n}+1$ satisfying the following conditions.

$$
\left\{\begin{array}{c}
L_{n}\left(f, z_{k}\right)=\alpha_{k} \quad, k=0,2 n+1 \\
L_{n}^{\prime}\left(f, z_{k}\right)=\beta_{k} \quad, k=1(1) 2 n
\end{array}\right.
$$

where, $\alpha_{k}$ and $\beta_{k}$ are arbitrary constants.

We establish convergence theorem for the same.

Theorem 3.1. $L_{n}(z)$ is regular on $Z_{n}$.

Proof: It is sufficient,if we show the unique solution of (3.1) is $L_{n}(z) \equiv 0$

In this case, let us consider

$$
\begin{aligned}
& \quad L_{n}^{\prime}\left(z_{k}\right)=0 \quad k=1(1) 2 n \\
& L_{n}^{\prime}(z)=a W(z) \\
& L_{n}(z)=a \int_{-1}^{z} W(t) d t
\end{aligned}
$$

Now, satisfying conditions in (3.1) we have, 
Convergence of Interpolatory Polynomial Between Lagrange and Hermite

$$
L_{n}(1)=a \int_{-1}^{1} W(t) d t=0
$$

As, $\int_{-1}^{1} W(t) d t \neq 0$, which implies $a=0$.

So, $L_{n}(z) \equiv 0$. Hence the theorem follows.

\section{Explicit representation of interpolatory polynomials}

We shall write,

$$
L_{n}(z)=\sum_{k=0,2 n+1} \propto_{k} A_{k}(z)+\sum_{k=1}^{2 n} \beta_{k} B_{k}(z)
$$

where, $A_{k}(z)$ and $B_{k}(z)$ are unique polynomial, each of degree atmost $2 n+1$ satisfying the conditions,

$$
\begin{gathered}
\left\{\begin{array}{cc}
A_{k}\left(z_{j}\right)=\delta_{k j} & j, k=0,2 n+1 \\
A_{k}^{\prime}\left(z_{j}\right)=0 & k=0,2 n+1 \quad j=1(1) 2 n
\end{array}\right. \\
\left\{\begin{array}{cc}
B_{k}\left(z_{j}\right)=0 \\
B_{k}^{\prime}\left(z_{j}\right)=\delta_{k j} \quad j, k=1(1) 2 n
\end{array}, k=1(1) 2 n\right.
\end{gathered}
$$

Theorem 4.1. For $k=1(1) 2 n$, we have

$$
\begin{aligned}
B_{k}(z)= & \left(z^{2}-1\right) L_{k}(z)-\int_{-1}^{z}\left(t^{2}-1\right) L_{k}^{\prime}(t) d t+ \\
& \left(1-2 z_{k}\right) \int_{-1}^{z} L_{k}(t) d t-S_{k}(1) \frac{\int_{-1}^{z} W(t) d t}{\int_{-1}^{1} W(t) d t}
\end{aligned}
$$

Proof: Let, $B_{k}(z)=\left(z^{2}-1\right) L_{k}(z)+S_{k}(z)+a \int_{-1}^{z} W(t) d t$

where, $B_{k}(z)$ is atmost of degree $2 n+1$ satisfying the conditions given in (4.3) provides us with

$$
a=-\frac{S_{k}(1)}{\int_{-1}^{1} W(t) d t}
$$

Applying the conditions given in (4.3) for $B_{k}{ }^{\prime}(z)$, we have

$$
S_{k}(z)=-\int_{-1}^{z}\left(t^{2}-1\right) L_{k}^{\prime}(t) d t+a_{k} \int_{-1}^{z} L_{k}(t) d t
$$

where, $a_{k}=1-2 z_{k}$

Hence, we have theorem 4.1.

Theorem 4.2. For $k=0,2 n+1$

$$
A_{k}(z)=(-1)^{k}\left(\frac{\int_{-z_{k}}^{z} W(t) d t}{\int_{-1}^{1} W(t) d t}\right)
$$

Proof: Let $A_{k}(z)=(-1)^{k} a_{k} \int_{-z_{k}}^{z} W(t) d t$

where, $A_{k}(z)$ is atmost of degree $2 n+1$ satisfying the conditions given in (4.2), we get

Hence the theorem follows.

$$
a_{k}=\frac{1}{\int_{-1}^{1} W(t) d t}
$$


Swarnima Bahadur and Varun

\section{Estimation of fundamental polynomials}

Lemma 5.1. Let $B_{k}(z)$ be given by (4.4), then

where, $c$ is a constant independent of $z$.

$$
\sum_{k=1}^{2 n}\left|B_{k}(z)\right| \leq \operatorname{clog} n
$$

Proof: Let $\quad \sum_{k=1}^{2 n}\left|B_{k}(z)\right| \leq I_{1}+I_{2}+I_{3}$

where, $I_{1}=\sum_{k=1}^{2 n}\left|\left(z^{2}-1\right) L_{k}(z)\right|$

Using (2.2), (2.7), (2.8), (2.9) and (2.11), we have

$$
I_{1} \leq \operatorname{clog} n
$$

Now, $\quad I_{2}=\sum_{k=1}^{2 n} \mid-\int_{-1}^{z}\left(t^{2}-1\right) L_{k}^{\prime}(t) d t+\left(1-2 z_{k}\right) \int_{-1}^{z} L_{k}(t) d t$

$$
I_{2} \leq \sum_{k=1}^{2 n} \int_{-1}^{z}\left|\left(t^{2}-1\right)\right|\left|L_{k}{ }^{\prime}(t)\right| d t+\left(1+2\left|z_{k}\right|\right) \int_{-1}^{z}\left|L_{k}(t)\right| d t
$$

Using (2.2), (2.7), (2.8), (2.9), (2.10) and (2.11), we have

$$
I_{2} \leq \text { clogn }
$$

Now, $\quad I_{3}=\sum_{k=1}^{2 n}\left|S_{k}(1) \frac{\int_{-1}^{z} W(t) d t}{\int_{-1}^{1} W(t) d t}\right|$

Using (2.2) and (2.10), we have

$$
I_{3} \leq c
$$

Combining (5.1), (5.2) and (5.3), our desired lemma follows.

Lemma 5.2. Let $A_{k}(z)$ be given in theorem (4.2), then

$$
\sum_{k=0,2 n+1}\left|A_{k}(z)\right| \leq c
$$

where, $\mathrm{c}$ is a constant independent of $z$ and $n$.

Proof: $\sum_{k=0,2 n+1}\left|A_{k}(z)\right|=\left|A_{0}(z)\right|+\left|A_{2 n+1}(z)\right|$

$$
\left|A_{0}(z)\right| \leq\left(\frac{1}{\left|\int_{-1}^{1} W(t) d t\right|}\right)\left|\int_{-1}^{z} W(t) d t\right|
$$

Using (2.2) and (2.10), we have

Similarly,

$$
\left|A_{0}(z)\right| \leq c
$$

$$
\left|A_{2 n+1}(z)\right| \leq c
$$

Combining these two equations, we get lemma 5.2.

\section{Convergence}

Theorem 6.1. Let $f(z)$ be continuous for $|z| \leq 1$ and analytic for $|z|<1$. Let the arbitrary $\beta_{k}$ 's be such that

$$
\left|\beta_{k}\right|=O\left(n \omega_{2}\left(f, \frac{1}{n}\right)\right) k=1(1) 2 n
$$

Then $\left\{L_{n}(z)\right\}$ defined by

satisfies the relation,

$$
L_{n}(z)=\sum_{k=0,2 n+1} f\left(z_{k}\right) A_{k}(z)+\sum_{k=1}^{2 n} \beta_{k} B_{k}(z)
$$

$$
\left|L_{n}(z)-f(z)\right|=O\left(\omega_{2}\left(f, n^{-1}\right) \log n\right),
$$


Convergence of Interpolatory Polynomial Between Lagrange and Hermite where $\omega_{2}\left(f, n^{-1}\right)$ be the modulus of continuity of second kind of $f(z)$.

Remark 6.1. Let $f(z)$ be continuous for $|z| \leq 1$ and analytic for $|z|<1$ and $f^{\prime} \varepsilon$ Lip $\alpha$, $\alpha>0$, then the sequence $\left\{L_{n}(z)\right\}$ converges uniformly to $f(z)$ in $|z| \leq 1$, which follows from (6.3) as

$$
\omega_{2}\left(f, n^{-1}\right) \leq n^{-1} \omega_{2}\left(f^{\prime}, n^{-1}\right)=O\left(n^{-1-\alpha}\right),
$$

To prove the theorem (6.1), we shall need following

Let $f(z)$ be continuous for $|z| \leq 1$ and analytic for $|z|<1$. Then thereexists a polynomial $F_{n}(z)$ of degree $\leq 2 n+1$ satisfying Jackson's inequality.

$$
\left|f(z)-F_{n}(z)\right| \leq c \omega_{2}\left(f, n^{-1}\right), \quad z=e^{i \theta}(0 \leq \theta<2 \pi)
$$
and also an inequality due to Kîs [3].

$$
\left|F_{n}^{(m)}(z)\right| \leq c n^{m} \omega_{2}\left(f, n^{-1}\right), m \varepsilon I^{+} \text {, where } c \text { is a constant. }
$$

Proof: Since, $L_{n}(z)$ be the uniquely determined polynomial of degree $\leq 2 n+1$ and the polynomial $F_{n}(z)$ satisfying (6.5) and (6.6) can be expressed as

$$
\begin{gathered}
F_{n}(z)=\sum_{k=0,2 n+1} F_{n}\left(z_{k}\right) A_{k}(z)+\sum_{k=1}^{2 n} F_{n}^{\prime}\left(z_{k}\right) B_{k}(z) \\
\left|L_{n}(z)-f(z)\right| \leq\left|L_{n}(z)-F_{n}(z)\right|+\left|F_{n}(z)-f(z)\right| \\
\leq \sum_{k=0,2 n+1}^{2 n}\left|f\left(z_{k}\right)-F_{n}\left(z_{k}\right)\right|\left|A_{k}(z)\right| \\
+\sum_{k=1}^{2 n}\left(\left|\beta_{k}\right|+\left|F_{n}^{\prime}\left(z_{k}\right)\right|\right)\left|B_{k}(z)\right| \\
+\left|F_{n}(z)-f(z)\right|
\end{gathered}
$$

Using (6.1), (6.4), (6.5), lemma 5.2 and lemma 5.3, we have the theorem 6.1.

\section{Conclusion}

In this paper, we have defined an intermediate interpolation problem between Lagrange and Hermite on some set of nodes on the unit circle and established a convergence theorem in same regard.

Acknowledgement. The authors are extremely thankful to the Editor-in-Chief and reviewers for their comments and suggestions for improving the paper.

\section{REFERENCES}

1. S.Bahadur, Convergence of Hermite interpolation, Global Journal of Theoretical and Applied Mathematical Science, 1(2) (2011)115-119.

2. S.Bahadur and Sariya Bano, Modified hermite interpolation on the unit circle, Annals of Pure and Applied Mathematics, 5(3) (2017) 531-537

3. S.Bahadur and K.K Mathur, Quasi-Hermite interpolation on the unit circle, Ganita, 49(1) (1998) 1-6.

4. L.Daruls and P.Gonzalezvera, A note on Hermite-Fejer interpolation for the unit circle, Applied Mathematics Letters, 14 (2001) 997-1003.

5. O.Kî̂, Remarks on interpolation (Russian), Acta Math. Acad. Sci. Hunger, 11(1960) 49-64. 
Swarnima Bahadur and Varun

6. J.Prasad, On Hermite and Hermite-Fejer interpolation of higher order, Demonstration Math., 26(2) (1993) 413-425.

7. T.L.Tu, On Hermite interpolation in roots of unity, Approximation, Optimization and Computing, (1990)197-200. 